\title{
Le feu des armes
}

\section{The fire of weapons}

\section{Roger Prud'homme ${ }^{1}$}

${ }^{1}$ Directeur de Recherches Emérite, Institut ə'Alembert, CNRS-Sorbonne-Université, Paris.

RÉSUMÉ. On fait dans ce court article un bilan sommaire des armes à base de combustion et de leurs effets. Sont évoqués les lance-flammes et l'arme thermobarique, les bombes : napalm, engins explosifs, bombes au phosphore. Puis il est question de missiles, d'armée de l'espace et de pollution due aux armes.

ABSTRACT. In this short article, we give a summary of combustion-based weapons and their effects. Flame throwers and thermobaric weapons, bombs: napalm, explosive devices, phosphorus bombs are mentioned. Then it comes to missiles, the space army and weapons pollution.

MOTS-CLÉS. combustion, armes, lance-flammes, bombes, explosions, armée de l'espace, pollution.

KEYWORDS. combustion, weapons, flamethrower, bombs, explosions, space army, pollution.

\section{Introduction}

Un projet d'article «Qu'est-ce que la combustion ? écrit sur proposition pour une encyclopédie avait fait réagir l'éditeur chargé de son suivi. Il trouvait le résumé de l'article trop neutre et pas assez motivant pour le lecteur et souhaitait l'améliorer.

Afin de montrer l'importance et l'intérêt du sujet, le texte suivant a été adopté dans OpenScience ${ }^{1}$ : «La découverte du feu a permis l'émergence de l'humanité. C'est ensuite la maîtrise scientifique de cette même combustion qui a permis à l'homme de se propulser sur la Lune, ou plus modestement de faire fonctionner efficacement le moteur de votre voiture. Qui n'a pas été fasciné par un feu d'artifice, une simple bougie ou un feu de bois? De nombreux phénomènes physiques et chimiques s'y produisent que cet article va vous permettre de découvrir. » L'article cité a été traduit pour un ouvrage en Anglais ${ }^{2}$.

Néanmoins, cette présentation de la découverte du feu ${ }^{3}$ et de ses conséquences peut paraître trop positive si l'on pense au feu des armes ${ }^{4}$, à la guerre, aux incendies provoqués par l'homme à des fins de destructions, aux armes perfectionnées et inhumaines utilisées souvent contre des civils et provoquant la mort et l'agonie dans des souffrances atroces. On dira que la dissuasion passe par là et qu'il faut bien se défendre. Mais il y a aussi les crimes contre l'humanité - notion récente, depuis la Seconde Guerre mondiale - qui marquent encore la réalité de notre temps. On sait que les luttes pour la paix - comme d'autres telles que les droits des femmes à la liberté, à la vie - sont des combats longs et ardus.

D'autre part bien des découvertes autres que le feu ont conduit à des recherches à finalités diverses, pas toutes caractérisées par des progrès humains. C'est aux sociétés d'œuvrer pour que les découvertes profitent réellement aux êtres humains.

\footnotetext{
${ }^{1}$ R. Prud'homme, Notions de base sur la combustion, Volume 19 - 1 DOI : $\underline{10.21494 / I S T E . O P .2019 .0376}$

${ }^{2}$ R. Prud'homme, Basics on interfaces in combustion, in Prud'homme R., Vincent S. (EDITS) - Fluid Mechanics at Interfaces - 1 methods and diversity, ISTE Series, (in production, 2021) - 150 pages. hal-03119319

${ }^{3}$ Il s'agit en fait de l'acquisition de la maîtrise du feu qui daterait d'environ 400000 ans.

${ }^{4}$ Jas Van Driel, consultant de la FESAC est l'auteur d'une histoire succincte des armes à feu publiée sur le site https://www.armes-ufa.com/spip.php?article39
} 
Il est bon de compléter la présentation des interfaces de combustion sous un autre aspect des activités humaines, tout comme on le fait à propos de cet autre problème de notre temps qu'est la pollution. Il est parfois utile de rappeler que les grandes découvertes n'ont pas toujours servi que des causes pacifiques. Ce fut le cas de la découverte du feu, de sa maitrise et de sa mise en œuvre par les humains. Des pertes en vies humaines ${ }^{5}$ en ont été et en sont toujours les conséquences, avec les problèmes de pollution qui s'ajoutent et sont rarement comptabilisés.

Il ne s'agit pas de mettre en cause ici l'existence d'une défense nationale, bien nécessaire tant que la paix mondiale n'existe pas, mais de souligner des conséquences dangereuses de l'agressivité guerrière tout en suggérant indirectement au lecteur d'œuvrer en faveur d'un monde pacifique.

Le présent article consiste principalement en une présentation des principales «armes à flammes », c'est-à-dire utilisant des flammes pour détruire des installations ou directement des êtres humains. Il est question ensuite des missiles puis de l'armée de l'espace et enfin de la pollution. On trouve finalement une conclusion comprenant des suggestions - on s'en doute - plutôt pacifistes, suivie de deux annexes. La première, A1, fait état de réflexions sur le sens de cet article. Les armes à feu classiques sont évoquées dans l'annexe A2, qui comprend d'autres part des compléments sur le napalm, le phosphore blanc et les Conventions de Genève.

\section{L'arme « flamme»}

\subsection{Le lance-flammes}

Le lance-flammes est une arme terrestre conçue pour projeter un liquide enflammé. Cette arme est soit manipulée par un fantassin, soit intégrée à l'armement d'un char.

L'effet létal est obtenu soit en arrosant directement la victime de liquide enflammé, soit en l'exposant à une forte chaleur, soit par consommation de l'oxygène dans un espace clos.

On compte aussi sur l'effet dissuasif de cette arme, par la terreur que provoque la perspective d'être brûlé vif.

L'arme peut également permettre d'incendier des bâtiments ou des véhicules.

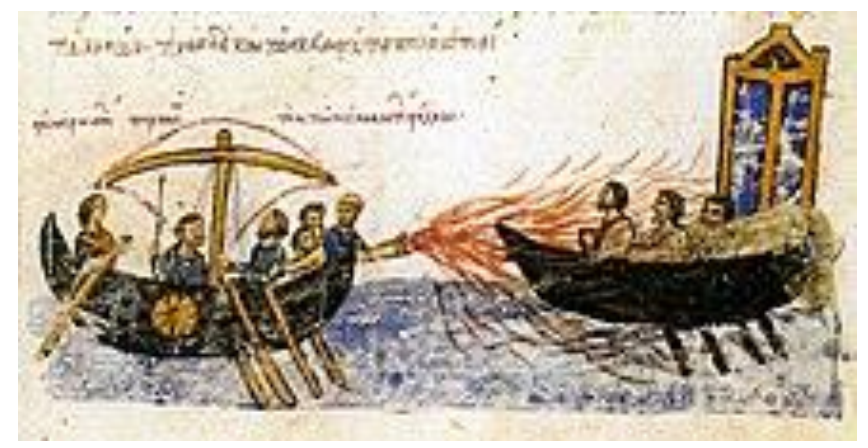

Figure 1. Image du XII siècle illustrant l'usage par la marine byzantine de feu grégeois contre la flotte rebelle de Thomas le Slave entre 820 et $823 .{ }^{6}$

\footnotetext{
${ }^{5}$ Il faut compter les conséquences des conflits armés, mais aussi celles des guerres civiles et celles de l'utilisation des armes en temps de paix (Rien qu'aux États-Unis en 2020, selon le site Gun Violence Archive, les armes à feu ont en effet fait plus de 43.000 morts, suicides inclus).

${ }^{6}$ https://fr.wikipedia.org/wiki/Thomas le Slave
} 
Cette arme a des prédécesseurs. L'un des plus anciens est le feu grégeois (Fig. 1). Le feu grégeois (du latin gracus, grec) est un mélange inflammable, brûlant même au contact de l'eau, employé, dans l'Antiquité et au Moyen Âge, pour la fabrication d'engins incendiaires utilisés au cours des sièges et des combats navals. Son invention est datée de la fin du VIIème et est attribuée à Callinicus d'Héliopolis, un architecte réfugié à Constantinople.

Un exemple de lance-flammes moderne est donné par la figure 2.

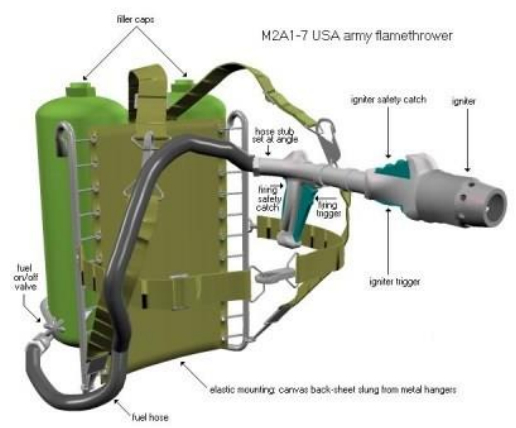

Figure 2. Le lance-flammes M2A1-7 de l'armée états-unienne.

Le lance-flammes est utilisé dans divers conflits durant la guerre froide dont ceux de Corée et du Vietnam où les forces américaines employèrent la dernière version de leur lance-flammes portable, le M9A1 dérivé du M2A1.

La dernière utilisation par l'armée française de cette arme a lieu en 1988 lors d'un assaut pendant la prise d'otage d'Ouvéa en Nouvelle-Calédonie ${ }^{7}$, pour neutraliser une mitrailleuse.

\subsection{L'arme thermobarique}

Délaissé depuis les années 1970/1980, le lance-flammes est remplacé dans la plupart des armées par les armes thermobariques (figure 3).

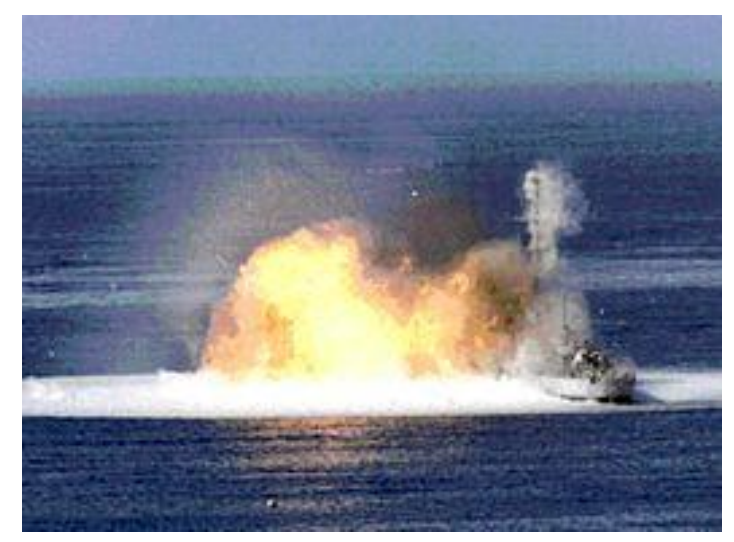

Figure 3. Tir d'un fuel air explosive de l'US Navy contre un navire cible, l'USS McNulty (en), en $1972 .^{8}$

\footnotetext{
${ }^{7}$ La prise d'otages d'Ouvéa, du $\underline{22}$ avril au $\underline{5} \underline{\text { mai }} \underline{1988}$, est le point culminant d'une succession d'événements violents survenus sur l'île d'Ouvéa (Nouvelle-Calédonie), et qui ont durablement marqué le territoire. Lire :

https://fr.wikipedia.org/wiki/Prise d\%27otages d\%270uv\%C3\%A9a\#L'assaut de la grotte : responsabilit\%C3\%A9s politiques et militaires

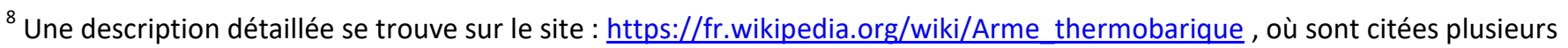
armes à explosifs thermobariques.
} 
Une arme thermobarique est une arme de type conventionnel, explosive, qui combine des effets thermiques, d'onde de choc et de dépression.

\section{Les bombes}

\subsection{Le napalm}

Dans l'armement le napalm ${ }^{9}$, inventé en 1942 , est de l'essence gélifiée, habituellement utilisée dans les bombes incendiaires. Sa formule est faite pour brûler à une température précise et coller aux objets et aux personnes (figures 4 et 5). En 1980, son usage contre les populations civiles a été interdit par une convention des Nations unies ainsi que par le Droit international humanitaire, prenant sa source dans les Conventions de Genève, qui proscrit toutes armes ne faisant pas la distinction entre les civils et les combattants.

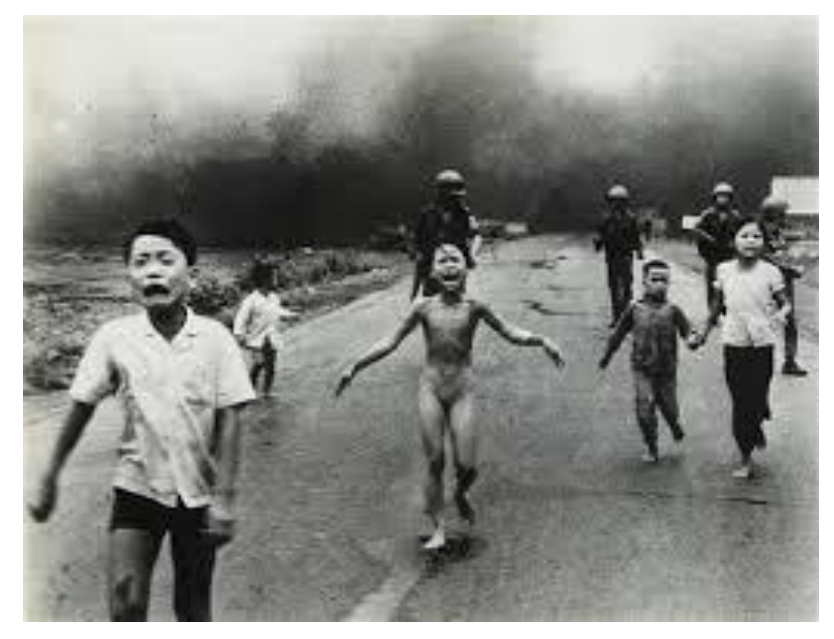

Figure 4. Les brûlures de la "petite fille au napalm » soignées 40 ans après. La photo de cette petite-fille brûlée après une attaque américaine au napalm pendant la guerre du Vietnam le 8 juin 1972 a fait le tour du monde. Plus de 40 ans après, Kim Phuc va enfin recevoir un traitement au laser pour soigner ses cicatrices. Artist: Nick Ut (Vietnamese, born 1951) Pulitzer Prize-winning photograph of children fleeing a napalm strike, South Vietnam, 1973. (source : google ${ }^{10}$ )

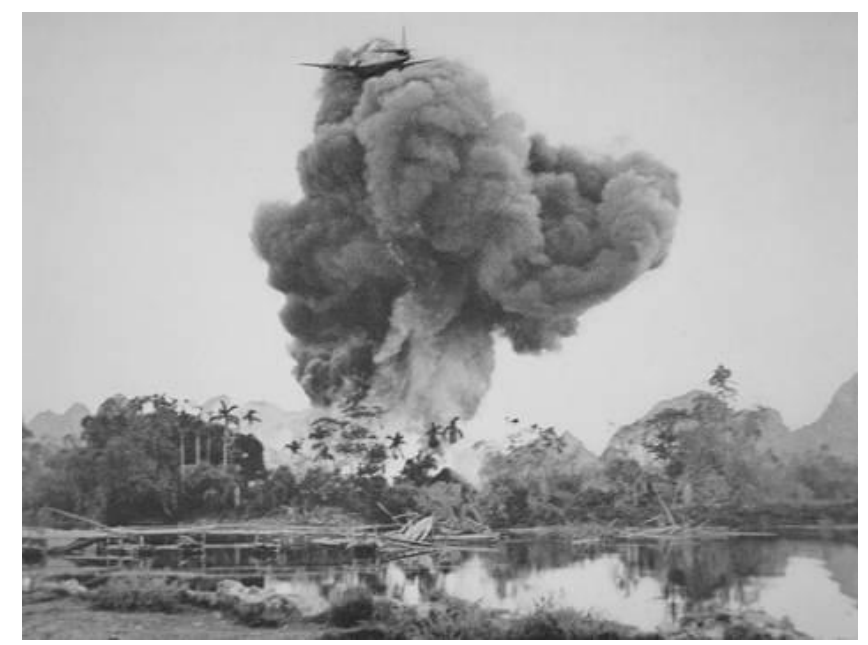

Figure 5. Bombardement français au napalm sur Phu Nho Quan, pendant la guerre d'Indochine en 1954.

\footnotetext{
${ }^{9}$ https://fr.wikipedia.org/wiki/Napalm

${ }^{10}$ https://www.google.com/search?q=napalm+au+vietnam\&hl=fr\&tbm=isch\&source=Int\&tbs=sur:fm\&sa=X\&ved=0ahUKEwig2s7 VxYjfAhUP1BoKHZSoCewQpwUIIA\&biw=1156\&bih=547\&dpr=1.5\#imgdii=nGQhFrgGzJj17M:\&imgrc=Oa_AKhI9I6TJHM
} 


\subsection{Les engins explosifs}

La force de frappe, le pilonnage des obus, le bruit, le feu. Pendant la Première Guerre mondiale, ce sont plus de 856 millions d'obus qui ont été tirés laissant derrière eux des paysages ravagés et des hommes traumatisés. (Source : Mission centenaire 14-18 ${ }^{11}$ ).

La Seconde Guerre mondiale a connu des destructions massives mettant en jeu des obus et des bombes causant des combustions explosives d'origine chimique, mais aussi les combustions nucléaires.

Une bombe est un engin explosif (figure 6) consistant habituellement en un contenant empli de matériel explosif ayant pour but de causer une destruction, lorsque déclenchée. À partir du $\mathrm{XX}^{\mathrm{e}}$ siècle, le mot est surtout utilisé pour désigner des engins explosifs lancés d'avions.

De l'explosion résulte la création d'un front d'onde de pression. La vitesse de ce front d'onde détermine la classification des explosifs. Il existe deux grands groupes d'explosifs :

- Les poudres ou explosifs soufflants (régime de déflagration). Ces explosifs peuvent aussi être utilisés pour la propulsion de projectiles ou fusées sous forme de poudre ou propergol solide.

- Les explosifs brisants (régime de détonation). Le plus puissant connu (l'octanitrocubane) atteint la vitesse de détonation de $10100 \mathrm{~m} / \mathrm{s}$. On peut citer parmi eux les groupes -nitros et nitrates, les peroxydes organiques, les chlorates et les perchlorates, les halogénures d'azote, les azotures et les fulminates.

La différence entre les régimes de déflagration et de détonation n'est pas toujours simple. Selon les conditions d'utilisation, un explosif normalement déflagrant peut détoner, et un explosif normalement détonant peut déflagrer. Les poudres sont conçues pour un régime de déflagration, c'est-à-dire une auto-combustion subsonique (la réaction chimique se propage à la vitesse de 10 à $400 \mathrm{~m} / \mathrm{s}$ dans l'explosif lui-même). Les explosifs progressifs se situent entre les poudres et les brisants. Ils suivent le régime de détonation supersonique (de 2000 à $3500 \mathrm{~m} / \mathrm{s}$ ). Les explosifs brisants détonent également (de 4000 à 9000 m/s). (Source : Explosif ${ }^{12}$ ).

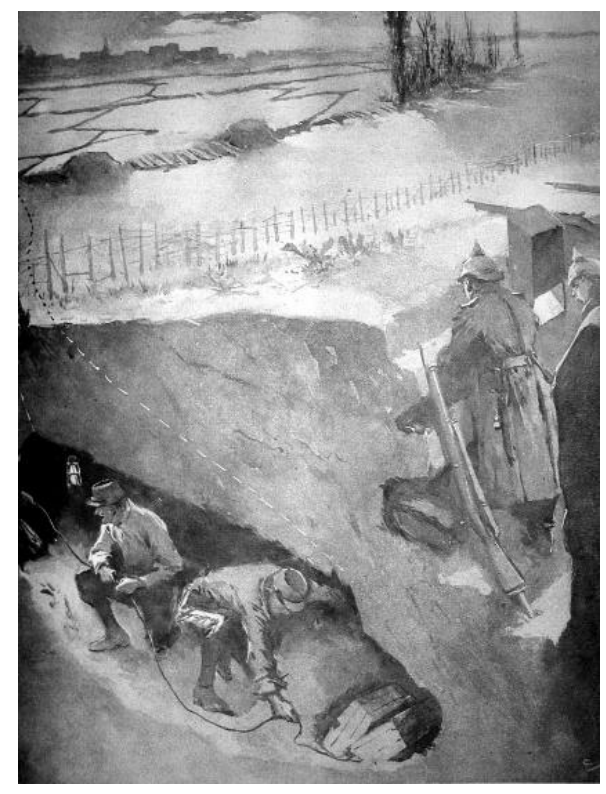

Figure 6. Utilisation d'explosifs par des sapeurs durant la Première Guerre mondiale. (source : Original téléversé par Boldair sur Wikipédia français).

\footnotetext{
${ }^{11}$ http://centenaire.org/fr/autour-de-la-grande-guerre/peinture/objets/explosions

${ }^{12}$ https://fr.wikipedia.org/wiki/Explosif
} 


\subsection{Les bombes au phosphore}

L'usage de ce type d'arme est dévastateur. Les particules incandescentes de phosphore blanc encore «affectueusement» appelé Willy Pete ${ }^{13}$ par les soldats britanniques lors de la Seconde Guerre mondiale et US au Viêt-Nam - pénètrent profondément dans la peau, jusqu'à faire fondre l'épiderme, les chairs et les os. Le phosphore blanc cause des brûlures chimiques multiples qui peuvent continuer à brûler dans le corps, même en l'absence d'oxygène extérieur. "Généralement, lorsqu'un patient présente une brûlure, on sait la soigner, et surtout il n'y a pas de détérioration, raconte le docteur Nafez Abou Shaaban, chef du service des brûlés à l'hôpital Shifa. Là, non seulement c'était impossible, mais en plus la plaie s'élargissait de plus en plus et, après quelques heures, de la fumée blanche s'en échappait. La seule solution que nous avions était d'amener le plus rapidement possible le patient en salle d'opération ${ }^{14}$. Ces brûlures sont souvent au deuxième et troisième degré.

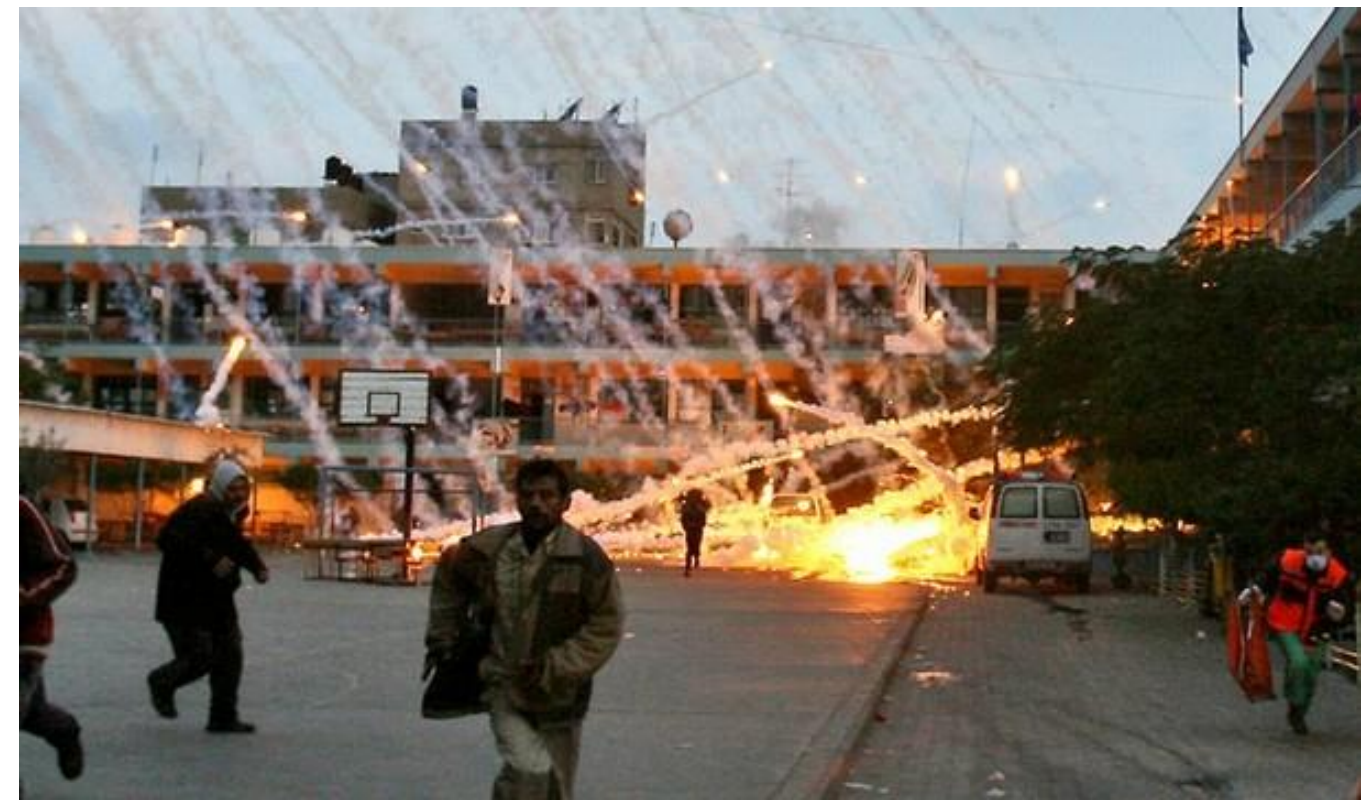

Figure 7. Gaza : pourquoi l'usage de bombes au phosphore blanc est un crime de guerre (Source : Basta ${ }^{15}$ ) Photos (@ lyad El-Baba) : bombardements au phosphore blanc de l'école de l'UNWRA

(administrée par les agences de l'ONU), à Beit Lahia, Gaza (2008-2009 opération “plomb durci »). L'attaque israélienne a tué deux enfants et mutilé leur mère et blessé des dizaines de personnes (y compris des enfants). Tous ceux qui ont respiré la fumée blanche chargée de particules toxiques vont en souffrir à plus long terme.

Au cours de son attaque contre Gaza, l'armée israélienne a fait usage de munitions au phosphore blanc (figure 7). Cette arme avait été utilisée par les États-Unis au Vietnam, par l'armée russe contre les Tchétchènes... ou par le dictateur Saddam Hussein contre les Kurdes irakiens. Malgré des dommages contre les populations similaires à ceux d'armes chimiques, le phosphore blanc est encore considéré par le droit international comme une «arme incendiaire » classique. En théorie.

\footnotetext{
${ }^{13}$ En anglais phosphore blanc se dit white phosphorus : WP.

${ }^{14}$ Le phosphore blanc brûle toujours, à l'hôpital de Gaza (article de Pierre Barbancey dans L'Humanité, 22-01-2009).

${ }^{15}$ https://www.bastamag.net/Gaza-pourquoi-l-usage-de-bombes-au
} 


\section{Les missiles}

Un missile est un projectile autopropulsé et guidé (sinon c'est une roquette), constitué de : un propulseur : moteur-fusée, réacteur (généralement statoréacteur ${ }^{16}$ ), voire les deux (une fusée donnant l'impulsion de départ, avant d'être relayée par un statoréacteur).

Un missile balistique se caractérise par sa trajectoire presque entièrement balistique, à savoir une trajectoire qui est influencée par la vitesse acquise lors de l'accélération initiale, la propulsion, et la gravité. L'engin peut envoyer une ou plusieurs armes.

Il y a actuellement une véritable course à la possession de missiles par les États. La portée est une caractéristique importante puisqu'elle détermine si un pays se trouve dans le champ d'action du missile.

La défense antimissile est l'ensemble des moyens mis en œuvre pour contrer la menace que représentent les missiles balistiques pour les forces armées sur les théâtres d'opérations et pour les populations sur les territoires nationaux. À son origine pendant la guerre froide, la défense antimissile vise à défendre les territoires américains et soviétiques contre les missiles balistiques intercontinentaux. Son important développement depuis le début du XXIe siècle est la conséquence de la prolifération des missiles balistiques, dans un contexte géopolitique marqué par les conflits au Proche-Orient et au Moyen-Orient, et par la montée des tensions en Asie et même en Europe. ${ }^{17}$

\section{La guerre de l'espace}

La protection des installations satellitaires incite les états les plus développés à créer des structures de défense.

La France vient de créer une section spéciale de la défense appelée armée de l'espace.

Gageons que cela ne conduira pas à une aggravation des conflits entre les États !

Là aussi la question des efforts de paix, du désarmement général et contrôlé sont plus que jamais décisives.

\section{Armes et pollution}

La revue Télescope a publié une étude très intéressante de Afifa Khazri, Professeure au Collège militaire royal du Canada sur la relation entre armes et pollution ${ }^{18}$. Dans le résumé de cet article, on peut lire :

«La relation entre les conflits sociaux, l'environnement et le développement durable est complexe et cette réalité doit être prise en compte dans toutes les décisions, économiques et politiques. Dans le présent article, nous étudions le lien entre le développement durable et les conflits armés. Nous croyons que pour parvenir à un tel développement les organismes internationaux doivent orienter leurs programmes de manière à sortir les pays pauvres de leur misère. »

Plusieurs sites web sont intéressants à consulter sur le sujet ${ }^{19}$.

\footnotetext{
${ }^{16}$ https://www.futura-sciences.com/sciences/definitions/univers-statoreacteur-2101/

${ }^{17}$ https://fr.wikipedia.org/wiki/D\%C3\%A9fense antimissile

${ }^{18}$ Khazri, A. (2011). « Le développement durable et les conflits armés », Télescope, vol. 17, n 2, p. 114-130.
} 
On y trouve des éléments : sur les produits toxiques dans les munitions laissés dans le sol à la suite des guerres; le très mauvais bilan carbone du transport aérien s'expliquant par l'origine militaire de celui-ci, qui aurait privilégié la puissance au rendement; les conflits armés et l'environnement (Cadre, modalités, méthodes et rôle de l'Évaluation environnementale) avec un article de Al-Hamandou Dorsouma et Michel-André Bouchard

[https://doi.org/10.4000/developpementdurable.3365]; un article de Jean-Marc Lavieille dans Médiapart portant sur l'ampleur des atteintes à l'environnement du fait des conflits armés; un article de Notre-planète - info sur les graves dommages des guerres sur l'environnement qui dénonce le recours massif à des produits hautement toxiques, le risque d'engrenage, les manipulations environnementales comme éléments de stratégie militaire, l'environnement comme cause de potentielle de guerre;

\section{Conclusion}

De nombreux ouvrages, des articles de presse et des spectacles ont montré les horreurs de la guerre.

Le contenu de cet article ne choquera donc pas le lecteur qui a pu lire ces ouvrages - dont «Guerre et Paix » de Léon Tolstoï -, ou assisté à la projection du film américain « Apocalypse Now » de Francis Ford Coppola .

Il s'agit ici de descriptions techniques ayant pour but de fournir une connaissance réaliste des armes mettant en jeu la combustion. Il ne s'agit pas de noircir la combustion plus que la suie ne le fait.

Il y a bien d'autres armes que les flammes ou les bombes à réactions chimiques. Les évènements du $20^{\text {ème }}$ siècle ont montré les dégâts des armes à feu classiques, des armes de destruction massive telles l'arme atomique - qui est aussi une combustion, mais pas classiquement chimique - au Japon, et ceux d'armes blanches - qui cependant ont aussi été forgées par le feu - au Rwanda.

Mais la vue des résultats des aventures guerrières peut inciter à les éviter au maximum.

Le contrôle du commerce des armes pourrait en ce sens être un facteur favorable, à condition que les pays acceptent de s'y soumettre (lire à ce sujet l'article de Tony Fortin ${ }^{20}$ ).

\section{Annexes}

\section{A1. Réflexions sur le contexte et le sens de cet article}

Le feu des armes. Faut-il se justifier avant d'aborder de telles questions ? Questions tabou ?

C'est peut-être surtout un problème de morale. En montrant la mort, les souffrances des individus causées par certaines armes, il y a une volonté d'inciter les sociétés à éviter de recourir à de tels procédés, de mettre en garde contre la cruauté et la perversité. Mais quelles sont les limites de la dissuasion?

\footnotetext{
${ }^{19}$ https://fr.wikipedia.org/wiki/Pollution induite par les munitions; https://reporterre.net/L-avion-champion-de-la-pollutionMerci-l-armee; https://journals.openedition.org/developpementdurable/3365 ;

https://blogs.mediapart.fr/lavieille/blog/290415/conflits-armes-lampleur-des-atteintes-lenvironnement-i ; https://www.notreplanete.info/actualites/3548-guerre environnement;

${ }^{20}$ Fortin, T. (2021). "Contrôle des ventes d'armes : quel rôle pour les parlementaires ?". Observatoire des armements. http://obsarm.org/spip.php?article353 ;
} 
En écrivant ainsi sur la feu des armes, risque-t-on de culpabiliser, non seulement les acteurs que sont les combattants, mais aussi ceux qui préparent les moyens des guerres? Est-ce juste ? Si oui, il ne faut pas se limiter à cela.

On pourrait d'ailleurs se poser des questions analogues à propos des conséquences d'actions policières (problème intérieur de chaque pays), de la pollution (international), des inégalités sociales (intérieur et international).

Cela ne soulève pas seulement des questions individuelles : il s'agit de problèmes de société, de problèmes politiques, économiques, commerciaux, qui interrogent aussi la pensée philosophique.

Il est normal de se poser ces problèmes, quel que soit notre situation citoyenne ou professionnelle dans la société. En ce sens cela concerne aussi les scientifiques à tous les niveaux.

Nous avons un devoir de vérité. Aussi l'information est nécessaire sur tous les plans, on le voit bien actuellement avec la pandémie de la covid !

\section{A2. Compléments}

\section{Sur les armes à feu classiques}

Une nombreuse documentation est disponible sur les armes à feu classiques ${ }^{21}$.

Par définition une arme à feu vise à toucher une cible éloignée via des projectiles (boulet, balle, bille ...), propulsés dans le canon de l'arme par des gaz en déflagration, issus de la combustion confinée d'un mélange chimique. La vitesse de combustion détermine de façon directe la vitesse à la «bouche ${ }^{22}$, dont dépend l'efficacité donc la portée. Le fait qu'en fonctionnement normal il s'agisse d'une déflagration, limite la vitesse d'éjection des gaz brûlés, donc celle du projectile, à environ $2000 \mathrm{~m} / \mathrm{s}$.

Dans les armes à feu modernes, les projectiles contiennent leur propre mélange déflagrant, enfermé dans le fond d'un étui ou d'une douille. Sa déflagration est déclenchée par un choc brutal produit par un percuteur ou par une décharge électrique.

Les armes à feu - où le feu est enfermé dans une chambre de combustion - sont apparues postérieurement au feu grégeois des byzantins, au XIIIème siècle (elles étaient utilisées par les troupes de Gengis Khan, puis par les Chinois, et en Europe dans la seconde moitié du Moyen Âge). A partir du XVII ${ }^{\mathrm{e}}$ siècle, le développement d'armes à feu individuelles fiables, réutilisables et peu coûteuses permet à l'Europe de dominer militairement le monde. Ces armes se sont généralisées dans tous les pays au cours des $\mathrm{XIX}^{\mathrm{e}}$ siècle et $\mathrm{XX}^{\mathrm{e}}$ siècle.

On peut classer deux grands types d'armes à feu :

1. les armes légères, armes individuelles, d'un calibre réduit, souvent en dessous de quinze millimètres, que l'on pointe à la main vers une cible visible ;

2. les armes lourdes, armes collectives, pièces d'artillerie, plus imposantes et nécessitant l'utilisation d'un affût pour être mises en batterie.

Mais on les classe aussi selon l'automatisation de leur séquence de tir. Elles sont utilisées pour la chasse, certains sports, le crime et la guerre. Parmi les multiples sortes d'arme à feu on trouve au cours des âges : l'arquebuse, l'arme à feu sous-marine, le bâton à feu, la carabine, le fusil

\footnotetext{
${ }^{21}$ L'on pourra consulter utilement le site https://fr.wikipedia.org/wiki/Arme \%C3\%A0 feu dont s'est inspiré, de façon très résumée, le présent paragraphe.

${ }^{22}$ La bouche du canon d'une arme est son extrémité la plus proche de la cible.
} 
(comprenant des fusils divers : d'assaut, de chasse, mitrailleur, à pompe, à poudre noire de précision), le lance-roquette, la mitrailleuse, le mousquet, le pistolet, le pistolet mitrailleur, la poivrière, le révolver, le tromblon ${ }^{23}$.

Les armes légères et de petit calibre sont considérées par l'ONU comme une source potentielle ou avérée d'exacerbation de la violence.

Mi-2007, un rapport établi par l'Institut universitaire de hautes études internationales de Genève estimait que 650 des 850 millions de petites armes à feu sur Terre $(76,5 \%)$ sont en fait détenues par des civils, le reste l'étant par les diverses armées et forces de l'ordre.

L'usage de certaines munitions en pleine nature ou dans les champs et prairies contribue de manière non négligeable à la pollution de l'environnement; plusieurs dizaines de milliers de tonnes de plomb sont ainsi dispersées dans l'environnement chaque année par la chasse et le ball-trap, qui se surajoutent aux apports des années précédentes de certaines pollutions diffuses de l'environnement (plomb toxique des balles ou de la grenaille, métaux lourds des amorces et de fumées de tir qui sont diffusés dans l'environnement, déchet toxique en quantité dispersée (DTQD) étant notamment source de saturnisme animal, aviaire en particulier et de saturnisme humain).

\section{Sur le napalm}

Le napalm cause de graves brûlures sur les personnes exposées ${ }^{19}$, par ses projections enflammées. Sa texture de gel colle à la peau et brûle les tissus jusqu'à l'os sans qu'il soit possible de stopper sa combustion. Il est illusoire de vouloir refroidir les plaies avec de l'eau.

En plus de sa puissance létale, le napalm est connu pour l'impact psychologique important qu'il exerce sur ses victimes.

En 1980, l'usage du napalm contre les populations civiles a été interdit par une convention des Nations Unies ainsi que par le Droit international humanitaire, prenant sa source dans les Conventions de Genève, qui proscrit toutes armes ne faisant pas la distinction entre les civils et les combattants.

\section{Sur le phosphore blanc}

De plus, le napalm est souvent combiné au phosphore blanc qui amplifie ses effets. Celui-ci permet d'enflammer le napalm et sert de détonateur dans ce type de bombe incendiaire. Lorsqu'une bombe au napalm explose au sol, le phosphore s'enflamme en premier à haute température, en laissant une traînée blanche caractéristique, puis le napalm s'enflamme. Il en résulte une grande boule de feu qui progresse rapidement et qui atteint la taille d'un immeuble de plusieurs étages pour les plus grosses munitions incendiaires.

Le phosphore blanc (aussi appelé phosphore jaune) est un solide blanc extrêmement inflammable et aux vapeurs corrosives.

Après la phase de combustion qui le transforme en pentoxyde de phosphore $\left(\mathrm{P}_{4} \mathrm{O}_{10}\right)$, il absorbe l'humidité de l'air et des tissus vivants pour former de l'acide phosphorique $\left(\mathrm{P}_{4} \mathrm{O}_{10}+6 \mathrm{H}_{2} \mathrm{O} \rightarrow 4\right.$ $\mathrm{H}_{3} \mathrm{PO}_{4}$ ), il en résulte (lors de l'utilisation de bombes), une dissolution des tissus vivants (composés majoritairement d'eau), mais aucun dégât aux vêtements.

\footnotetext{
${ }^{23}$ Il est utile de définir certaines de ces armes anciennes, par exemple : le mousquet est une arme à feu portative au long canon à âme lisse, crosse d'épaule et platine à mèche ou à rouet, en usage dans l'infanterie aux $\mathrm{XVI}^{\mathrm{e}}$ et XVII $^{\mathrm{e}}$ siècles, la poivrière est un type d'arme à feu à canons tournants, sorte de revolver avec un barillet très allongé dont chacune des quatre à six chambres (parfois huit, voire plus) fait directement office de canon.
} 
Il peut être transformé en phosphore rouge, un allotrope amorphe du phosphore inoffensif, en le chauffant vers $250{ }^{\circ} \mathrm{C}$, car le phosphore rouge est l'allotrope thermodynamiquement stable aux conditions normales de température et de pression.

En 1983, l'ONU interdit son utilisation offensive et classe son utilisation en crime de guerre ${ }^{24}$.

Israël a utilisé le phosphore blanc comme fumigène, lors du siège de Beyrouth en 1982, pendant l'offensive sur le Liban de 2006 et dans la bande de Gaza à l'automne 2006, ainsi que durant la guerre de Gaza 2008-2009 avec des obus éclairants sur les zones urbaines de la bande de Gaza. L'armée israélienne assure que l'utilisation de ces armes «s'exerce dans le cadre des frontières légales du droit international ». Le Hamas a lancé des bombes au phosphore depuis la bande de Gaza vers Israël en 2010.

À cette occasion le Comité international de la Croix-Rouge a donné un aperçu des règles applicables aux armes au phosphore et expliqué la position du CICR, sans confirmer l'utilisation d'armes au phosphore blanc par Israël à Gaza. L'organisation humanitaire Human Rights Watch considère cependant que le "droit international coutumier" interdit l'utilisation des armes au phosphore blanc dans un endroit aussi densément peuplé que la bande de Gaza.

\section{Sur les conventions de Genève}

Les conventions de Genève sont des traités internationaux fondamentaux dans le domaine du droit international humanitaire. Elles dictent les règles de conduite à adopter en période de conflits armés, et notamment la protection des civils, des membres de l'aide humanitaire, des blessés ou encore, des prisonniers de guerre.

La première convention de Genève date de 1864. Cependant, les textes en vigueur aujourd'hui ont été écrits après la Seconde Guerre mondiale.

Sept textes ont cours actuellement : les quatre conventions de Genève du 12 août 1949, les deux protocoles additionnels du 8 juin 1977 et le troisième protocole additionnel de 2005. Les quatre conventions de Genève ont été mondialement ratifiées, ce qui signifie que chacun des États du monde s'engage à les respecter.

\footnotetext{
${ }^{24}$ https://fr.wikipedia.org/wiki/Phosphore blanc , https://www.francetvinfo.fr/monde/proche-orient/israel-palestine/israel-achaque-offensive-de-tsahal-son-arme-polemique_652919.html
} 